\title{
Validación psicométrica del POSIT para el cribado del consumo de riesgo de alcohol y otras drogas entre adolescentes
}

\section{Psychometric validation of the POSIT for screening alcohol and other drugs risk consumption among adolescents}

\author{
Manuel Araujo*, Sandra Golpe*, Teresa Braña*, Jesús Varela*, Antonio Rial*. \\ * Universidad de Santiago de Compostela, España.
}

\section{Resumen}

La detección precoz del consumo abusivo de alcohol y otras drogas en adolescentes resulta decisiva no sólo para una derivación e intervención rápida en los casos de riesgo, sino también como un indicador a utilizar en la evaluación de los programas de prevención y en las políticas públicas de reducción del consumo. Uno de los instrumentos de screening más utilizados a nivel internacional es el Problem Oriented Screening Instrument for Teenagers (POSIT) (Rahdert, 1991), cuya subescala de Uso y Abuso de Sustancias ( POSIT $_{\text {UAS }}$ ) constituye una herramienta breve de enorme potencial aplicado. Sin embargo, en España no existe todavía ningún estudio de validación empírica que permita garantizar su adecuado funcionamiento psicométrico. El objetivo del presente trabajo consiste precisamente en analizar las propiedades psicométricas del POSIT $_{\mathrm{UAS}}$ en adolescentes españoles. Para ello fueron entrevistados de forma personal 569 estudiantes de entre 12 y 18 años $(M=14,71 ; D T=1,79)$, seleccionados a partir de un muestreo bietápico. Los resultados obtenidos, utilizando la Adolescent Diagnostic Interview (Winters \& Henly, 1993) como criterio, permiten informar que la versión española del POSIT $_{\text {UAS }}$ posee un excelente comportamiento psicométrico, tanto a nivel de consistencia interna $(a=82)$, como de sensibilidad (94,3\%) y especificidad (83,9\%), con un área bajo la curva ROC de ,953. Asimismo, la realización de un Análisis Factorial Confirmatorio permite constatar el carácter unidimensional de la escala. Como consecuencia se pone a disposición de investigadores y profesionales del ámbito de las conductas adictivas el $\operatorname{POSIT}_{\text {UAS }}$, que puede ser utilizado en adelante con las garantías psicométricas requeridas.

Palabras clave: Adolescentes; Alcohol; Cribado; Drogas; POSIT.

\begin{abstract}
Early detection of alcohol and drug abuse among adolescents is decisive not only for rapid referral and intervention in cases of risk, but also as an indicator for use in the evaluation of prevention programs and public policies to reduce consumption. One of the most widely-used screening instruments in the world is the Problem Oriented Screening Instrument for Teenagers (POSIT) (Rahdert, 1991), whose substance use and abuse subscale $\left(\right.$ POSIT $\left._{\text {UAS }}\right)$ is a brief tool of enormous applied potential. However, there is still no empirical validation study that would ensure its good psychometric performance in Spain. The aim of this paper is to analyse the psychometric properties of POSIT $_{\mathrm{UAS}}$ among Spanish adolescents. For this purpose, 569 students aged between 12 and 18 years $(M=14.71 ; S D=1.79)$ were personally interviewed. The study sample was selected through two-stage sampling. The results obtained, using the Adolescent Diagnostic Interview (Winters \& Henly, 1993) as the gold criterion, allow us to inform that the Spanish version of the POSIT $_{\text {UAS }}$ has excellent psychometric behaviour, both at the level of internal consistency $(\mathrm{a}=.82)$ as well as regards sensitivity $(94.3 \%)$ and specificity $(83.9 \%)$, with an area under the ROC curve of .953. Also, the realisation of a Confirmatory Factor Analysis allows for verifying the one-dimensional character of the scale. As a result, POSIT $_{\text {UAS }}$ is made available to researchers and professionals in the field of addictive behaviours for use with a minimum of psychometric guarantees.
\end{abstract}

Key words: Adolescents; Alcohol; Screening; Drugs; POSIT. 
$\mathrm{E}$ 1 consumo de alcohol y otras sustancias entre los jóvenes constituye uno de los principales problemas sociosanitarios en España. A pesar de que los datos recogidos en la última Encuesta Estatal sobre uso de Drogas en Enseñanzas Secundarias [ESTUDES 2014-2015] (Plan Nacional sobre Drogas, 2016) señalan que los niveles de consumo de las diferentes sustancias han disminuido en los últimos años, las cifras de prevalencia siguen siendo elevadas. Un 76,8\% de los estudiantes entre 14 y 18 años consumieron alcohol en el último año (68,2\% en el último mes); un $31,4 \%$ indicaron haber fumado tabaco en el último año (25,9\% en el último mes) y el 25,4\% reconocieron haber consumido cannabis $(18,6 \%$ en el último mes). El resto de sustancias exploradas tales como la cocaína, el éxtasis, las anfetaminas o los alucinógenos presentan cifras de prevalencia mucho menores, por debajo del $3 \%$.

De entre todas las sustancias analizadas en el ESTUDES 2014-2015, el alcohol sigue siendo la sustancia psicoactiva más consumida por los adolescentes. En este contexto, algunas de las cuestiones que más siguen preocupando a profesionales e investigadores son el consumo intensivo de alcohol, también conocido como binge drinking (Farke y Anderson, 2007; Parada et al., 2011), unido a la temprana edad a la que los adolescentes se inician en su consumo. Según el ESTUDES 2014-2015, el 32,2\% de los adolescentes practicaron binge drinking en el último mes y un $22,2 \%$ señalaron haberse emborrachado. Existe abundante literatura que pone de manifiesto que este patrón de consumo tan extendido a día de hoy entre los jóvenes conlleva no sólo a importantes consecuencias a nivel orgánico (López-Caneda et al., 2014), sino también una mayor probabilidad de verse involucrados en numerosas prácticas de riesgo (Huang, Jacobs y Deverensky, 2010; Matali et al., 2016; Miller, Naimi, Brewer y Jones, 2007; Windle, 2003) y de desarrollar un patrón de policonsumo o un posible trastorno/dependencia de alcohol en la edad adulta (Jones, Oeltmann, Wilson, Brener y Hill, 2001; Petit, Maurage, Kornreich, Verbanck y Campanella, 2014). Una variable que incide en la probabilidad de aparición de muchas de estas consecuencias y en su gravedad es la edad a la que se empieza a consumir alcohol (Motos, Cortés, Giménez y Cadaveira, 2015). Tanto es así que el retraso de la edad de inicio aparece contemplado como uno de los objetivos del Plan de Acción sobre Drogas 2013-16 (Plan Nacional sobre Drogas, 2009a) (objetivo general 4), así como en diferentes planes autonómicos, como el Plan de Trastorno Adictivos de Galicia 2011-2016 (Xunta de Galicia, 2010) (objetivo 1.3).

No menos preocupantes son las cifras de prevalencia de consumo de cannabis ya señaladas, lo que la convierte en la droga ilegal más consumida por parte de los adolescentes. Más allá de los niveles de consumo revelados por el ESTUDES 2014-15, cabe señalar que un 2,5\% (53701 adolescentes aproximadamente) llegaron incluso a ser identificados, a través del Cannabis Abuse Screening Test (CAST) (Legleye,
Piontek y Kraus, 2011), como consumidores problemáticos o en riesgo de desarrollar una posible dependencia (Plan Nacional sobre Drogas, 2016).

En este contexto, la detección precoz del consumo abusivo de alcohol y otras drogas en adolescentes resulta decisiva no sólo para favorecer una rápida derivación e intervención sobre los casos de riesgo, sino también como indicador a utilizar en la evaluación de los programas de prevención y en las políticas de reducción del consumo. Se hace necesario, por lo tanto, disponer de instrumentos de screening suficientemente contrastados a nivel internacional, debidamente traducidos y adaptados a nuestra cultura y, al mismo tiempo, con suficiente aval empírico-psicométrico (García, Novalbos, Martínez y O’Ferrall, 2016; Tiburcio et al., 2016).

Uno de los instrumentos que ha mostrado mayor utilidad en este ámbito ha sido el Problem Oriented Screening Instrument for Teenagers (POSIT). El POSIT se dio a conocer en una publicación del National Institute on Drug Abuse (Rahdert, 1991) como uno de los instrumentos que componían el Adolescent Assessment/Referral System (AARS) cuya elaboración y desarrollo había comenzado ya en 1987. Fue desarrollado con el fin de poder detectar oportunamente problemas específicos de los adolescentes que usan/abusan de las drogas. Consta de 139 ítems de respuesta dicotómica (sí/no) que se agrupan en 10 subescalas que evalúan las diferentes áreas de funcionamiento de la vida de los adolescentes que pueden verse afectadas por el uso de drogas, siendo la subescala de Uso y Abuso de Sustancias (POSI$\mathrm{T}_{\text {UAS }}$ ) una de ellas. Dicha subescala consta de 17 ítems que tienen como objetivo realizar un screening de adolescentes que podrían estar en riesgo de desarrollar un posible trastorno o dependencia por consumo de sustancias.

Actualmente existen diversos estudios sobre la validación y la utilidad del POSIT, tanto de la escala completa (Dembo et al., 1996; Knight, Goodman, Pulerwitz y DuRant, 2001; McLaney, Del Boca y Babor, 1994) como de algunas de sus subescalas (Knight, Sherritt, Harris, Gates y Chang, 2003; Latimer et al., 2004; Latimer, Winters y Stinchfield, 1997; Rumpf, Wohlert, Freyer-Adam, Grothues y Bischof, 2013). La utilidad del POSIT como herramienta de screening en adolescentes ha sido recalcada por diferentes autores e instituciones, tanto en revistas científicas como en guías y manuales, destacando la fiabilidad y validez del instrumento (Center for Substance Abuse Treatment, 2012; Fuller y Cavanaugh, 1995; McPherson y Hersch, 2000; Organización Mundial de la Salud, 1997; Timken, 2007).

Por lo que se refiere específicamente a la subescala de Uso y Abuso de Sustancias (POSIT ${ }_{\text {UAS }}$ ) son relativamente pocos los trabajos que hayan informado de sus propiedades psicométricas (Knight et al., 2001, 2003; Latimer et al., 2004, 1997), sin que exista hasta el día de hoy ningún trabajo de validación empírica realizado en España (Bobes et al., 2013). Por lo general los trabajos llevados a cabo en 
otros países sí han constatado, no obstante, unas excelentes propiedades psicométricas de la subescala, lo que cual le confiere una enorme potencialidad a nivel aplicado, habida cuenta de su brevedad y fácil aplicación.

En definitiva, a pesar de haberse convertido en una de las herramientas de evaluación y screening más utilizadas a nivel mundial, seguimos sin disponer de estudios empíricos de validación que permitan conocer cuál es realmente el comportamiento psicométrico de la subescala de Uso y Abuso de Sustancias (POSIT ${ }_{\text {UAS }}$ ) con adolescentes españoles. Este es precisamente el objetivo central del presente trabajo. De manera más concreta, las hipótesis que se intentarán poner a prueba en el estudio empírico son dos: en primer lugar, que el POSIT ${ }_{\mathrm{UAS}}$ constituye un instrumento adecuado a nivel psicométrico para detectar precozmente problemas de consumo de alcohol y/u otras sustancias en adolescentes españoles y, en segundo lugar, en lo que se refiere a la su validez interna o estructura factorial, se intentará verificar su carácter unidimensional.

\section{Método}

\section{Participantes}

Para dar cuenta del objetivo señalado se recurrió a una metodología selectiva consistente en la realización de entrevistas individuales a estudiantes de Educación Secundaria Obligatoria (ESO), Bachillerato y Ciclos Formativos de grado medio de la comunidad autónoma de Galicia (España). Para la selección de la muestra se recurrió a un muestreo bietápico: por conglomerados, para la selección de las unidades de primer nivel (centros educativos) y por cuotas, para la selección de las unidades de segundo nivel (individuos).

A pesar de que inicialmente fueron entrevistados un total de 600 adolescentes, la muestra final estuvo compuesta por 569 individuos, descartándose 31 fundamentalmente por no haber podido completar la entrevista en su totalidad o por incoherencias manifiestas en las respuestas. Para garantizar que no existía ningún sesgo en la distribución de los casos missingy que éstos se distribuían de forma aleatoria, se comprobó que el porcentaje de casos perdidos fuese similar en los diferentes segmentos muestrales en función del género, grupo de edad, titularidad del centro y entorno de residencia, calculando para ello estadísticos de contraste $\chi^{2}$.

Respecto a la composición de la muestra, el $57,1 \%$ eran hombres y el $42,9 \%$ mujeres y sus edades estaban comprendidas entre los 12 y los 18 años $(M=14,71 ; S D=1,79)$. Los participantes fueron seleccionados aleatoriamente de un total de 33 centros educativos (22 públicos y 11 privados). De estos, el 69,8\% se encontraban cursando la ESO $(34,8 \%$ en el primer ciclo y el $35 \%$ en el segundo), el 20,4\% en Bachillerato y el 9,8\% FP Básica o un Ciclo Formativo de grado medio. Por último, el 43,1\% residían en un entorno urbano y el $56,9 \%$ rural o semirural.

\section{Instrumento}

Los datos fueron recogidos mediante una entrevista estructurada, con el apoyo de un cuestionario en el que se incluían: la subescala de Uso y Abuso de Sustancias del Problem Oriented Screening Instrument for Teenagers (POSIT ${ }_{\text {UAS }}$ ) (Rahdert, 1991), la Adolescent Diagnostic Interview (ADI) (Winters y Henly, 1993) y el CRAFFT Substance Abuse Screening Test (Knight et al., 1999). Para evitar un posible sesgo del orden de cumplimentación de los tres instrumentos, éste fue debidamente contrabalanceado.

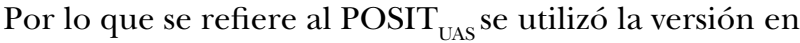
castellano facilitada por el European Monitoring Centre for Drugs and Drug Addiction (EMCDDA) (1998), que consta de 17 ítems dicotómicos (sí/no) y su puntuación teórica oscila entre 0 y 17. La Adolescent Diagnostic Interview (ADI) (Winters y Henly, 1993) fue utilizada como criterio para calcular la sensibilidad y especificidad del POSIT $_{\text {UAS }}$ y consiste en una entrevista diagnóstica de 213 ítems adaptada a los criterios del DSM-5 (American Psychiatric Association [APA], 2013), para la identificación de trastornos por consumo de sustancias en adolescentes. Para su empleo en este trabajo, dicha entrevista fue traducida y contra-traducida bajo la supervisión de sus autores originales. La consistencia interna obtenida para las distintas escalas diagnósticas fue elevada $(\alpha=, 84$ para el diagnóstico de un trastorno por consumo de alcohol; $\alpha=, 87$ para el diagnóstico de un trastorno por consumo de cannabis y $\alpha=$ ,88 para el diagnóstico de un trastorno por consumo de sustancias). Por último, como indicador complementario de validez de criterio se incluyó también el CRAFFT, escala compuesta de tan sólo 6 ítems y diseñada específicamente para el screening del consumo de riesgo de alcohol y otras sustancias en adolescentes. Fue traducida y contra-traducida bajo la supervisión de sus autores originales y validada empíricamente en el trabajo de Araujo et al. (2015), mostrando una consistencia interna ( $\alpha$ de Cronbach) de ,74, muy similar a la obtenida en el estudio de validación original de Knight, Sherritt, Shrier, Harris y Chang (2002) $(\alpha=, 72)$.

\section{Procedimiento}

Los datos fueron recogidos a través de una entrevista personal realizada en los propios centros educativos, en despachos habilitados para tal fin, por un equipo de psicólogos/as con experiencia en este tipo de trabajos. La duración de cada entrevista osciló entre 45 y 60 minutos. Los participantes fueron informados de la finalidad del estudio, así como del anonimato y la confidencialidad de sus respuestas. El estudio contó con el consentimiento y colaboración tanto de la dirección de los centros como de las respectivas asociaciones de padres/madres. La participación fue totalmente voluntaria y no remunerada. El estudio fue aprobado por el Comité Bioético de la Universidad de Santiago de Compostela. 


\section{Análisis de datos}

En primer lugar se realizó un análisis descriptivo mediante el cálculo de porcentajes, así como de los estadísticos de tendencia central y de dispersión. Además se realizaron comparaciones de medias en función del género (mediante la aplicación de la prueba $t$ de Student) y del grupo de edad (a través de un Anova unifactorial y un contraste post-hoc de Tukey). Para evaluar la consistencia interna se calculó el índice KR-20, adecuado para variables dicotómicas (Kuder y Richardson, 1937). Para verificar la estructura unidimensional de la escala se realizó un Análisis Factorial Confirmatorio (AFC) partiendo de la matriz de correlaciones tetracórica. Debido a la propia métrica de los datos y la ausencia de normalidad, para la estimación de parámetros se utilizó el método de Mínimos Cuadrados No Ponderados (ULS), que además de ser un método robusto no requiere ninguna suposición alguna acerca de su distribución (Jöreskog y Sörbom, 1989). La bondad de ajuste del modelo fue evaluada a través de los siguientes índices: el índice de bondad de ajuste (GFI [Goodness of Fit Index]), el índice de bondad de ajuste corregido (AGFI [Adjusted Goodness of Fit Index] ) y el índice de ajuste normalizado (NFI [Normed Fit Index]).

Para analizar las propiedades psicométricas de la escala se calcularon los índices de sensibilidad y especificidad y, de manera complementaria, el área bajo la curva ROC (Receiver's Operating Characteristics) con el objetivo de determinar el punto de corte óptimo. Por último, para evaluar la validez de criterio se analizó el grado de concordancia

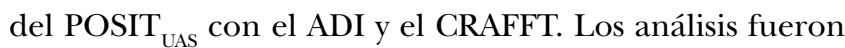
realizados con el paquete estadístico IBM SPSS Statistics 20 (IBM Corp. Released, 2011) y AMOS 21 (Arbuckle, 2012).

\section{Resultados}

\section{Estadísticos descriptivos}

En primer lugar, se presentan en la Tabla 1 las respuestas directas de los 569 adolescentes a cada uno de los 17 ítems que componen el POSIT $_{\text {UAS }}$, con el porcentaje de sujetos que respondieron afirmativamente a cada uno de ellos. Los mayores porcentajes corresponden al ítem 6 (“¿llevan tus amigos drogas a las fiestas?”) y al ítem 2 (“¿se aburren tus amigos en las fiestas donde no se sirven bebidas alcohólicas?”), con un 30,2\% y un 29,3\% de respuestas afirmativas, respectivamente. Por su parte, el ítem1 (“¿te metes en problemas porque consumes drogas o bebidas alcohólicas en la escuela?") y el ítem 10 (“¿has tenido un accidente de coche o moto estando bajo el efecto del alcohol o de las drogas"?) son los que presentan menor porcentaje de respuestas afirmativas $(0,5 \%$ y $0,7 \%$, respectivamente).

Tabla 1. Porcentaje de respuestas afirmativas a cada uno de los ítems del POSIT UAS, cargas factoriales estandarizadas y $p$-valor.

\begin{tabular}{|c|c|c|c|}
\hline Ítem & \% sí & $\lambda$ & $t$ \\
\hline ¿Te metes en problemas porque consumes drogas o bebidas alcohólicas en la escuela? & 0,5 &, 16 & $5,38^{\star \star \star}$ \\
\hline ¿Se aburren tus amigos en las fiestas donde no se sirven bebidas alcohólicas? & 29,3 & ,42 & $7,20^{\star \star \star}$ \\
\hline $\begin{array}{l}\text { ¿Te has hecho daño o le has hecho daño a otra persona accidentalmente estando bajo el efecto del alcohol o } \\
\text { las drogas? }\end{array}$ & 4,7 & ,47 & $10,08^{\star \star \star}$ \\
\hline $\begin{array}{l}\text { ¿A veces no puedes participar en actividades porque no tienes dinero por habértelo gastado en drogas o } \\
\text { bebidas alcohólicas? }\end{array}$ & 3,7 &, 41 & $10,34^{\star \star \star}$ \\
\hline ¿Sientes a veces que eres adicto/a al alcohol o a las drogas? & 3,7 & ,47 & $10,53^{\star \star \star}$ \\
\hline ¿Llevan tus amigos drogas a las fiestas? & 30,2 &, 60 & $9,43^{\star \star \star}$ \\
\hline ¿Has comenzado a consumir mayor cantidad de drogas o alcohol cada vez para obtener el efecto que deseas? & 7,6 &, 51 & $10,25^{\star \star \star}$ \\
\hline ¿Te vas a veces de las fiestas porque no hay bebidas alcohólicas o drogas? & 3 & ,46 & $9,64^{\star \star \star}$ \\
\hline ¿Sientes un deseo constante de consumir bebidas alcohólicas o drogas? & 1,2 &, 30 & $7,95^{\star \star \star}$ \\
\hline ¿Has tenido un accidente de coche o moto estando bajo el efecto del alcohol o de las drogas? & 0,7 &, 28 & $8,20^{\star \star \star}$ \\
\hline ¿Se te olvidan las cosas que hiciste mientras estabas consumiendo alcohol o drogas? & 13,9 &, 66 & $10,10^{\star \star \star}$ \\
\hline $\begin{array}{l}\text { ¿Te ocasiona el alcohol o las drogas cambios rápidos de estado de ánimo como pasar de estar alegre a estar } \\
\text { triste, o lo contrario? }\end{array}$ & 19,3 &, 67 & $10,03^{\star \star \star}$ \\
\hline $\begin{array}{l}\text { ¿Te han dicho alguna vez tus familiares o amigos que debes reducir el consumo de bebidas alcohólicas o } \\
\text { drogas? }\end{array}$ & 9,5 &, 57 & $10,68^{\star \star \star}$ \\
\hline $\begin{array}{l}\text { ¿Tienes discusiones serias con tus amigos o con miembros de tu familia por tu consumo de bebidas } \\
\text { alcohólicas o drogas? }\end{array}$ & 3 & ,36 & $8,99^{\star \star \star}$ \\
\hline $\begin{array}{l}\text { ¿Cuándo consumes bebidas alcohólicas o drogas, tienes a hacer cosas que normalmente no harías, tales } \\
\text { como desobedecer reglas, infringir leyes o llegar tarde a casa? }\end{array}$ & 16,9 &, 70 & $10,42^{\star \star \star}$ \\
\hline ¿Tienes dificultad en tus relaciones con alguno de tus amigos debido a tu consumo de alcohol o drogas? & 1,6 &, 25 & $6,23^{\star \star \star}$ \\
\hline ¿Sientes a veces que no puedes controlar el deseo de consumir bebidas alcohólicas o drogas? & 3,5 & ,42 & $7,95^{\star \star \star}$ \\
\hline
\end{tabular}

Nota. ${ }^{\star * \star} \mathrm{p}<, 001$. 
Tabla 2. Distribución de frecuencias para la puntuación total del POSIT UAS

\begin{tabular}{cccc}
\hline $\begin{array}{c}\text { Puntuación } \\
\text { Total }\end{array}$ & Frecuencia & $\begin{array}{c}\text { Porcentaje } \\
\text { válido }\end{array}$ & $\begin{array}{c}\text { Porcentaje } \\
\text { acumulado }\end{array}$ \\
\hline 0 & 288 & 50,6 & 50,6 \\
1 & 94 & 16,5 & 67,1 \\
2 & 61 & 10,7 & 77,9 \\
3 & 33 & 5,8 & 83,7 \\
4 & 35 & 6,2 & 89,8 \\
5 & 15 & 2,6 & 92,4 \\
6 & 15 & 2,6 & 95,1 \\
7 & 9 & 1,6 & 96,7 \\
8 & 9 & 1,6 & 98,2 \\
9 & 2 & 0,4 & 98,6 \\
10 & 3 & 0,5 & 99,1 \\
11 & 1 & 0,2 & 99,3 \\
12 & 2 & 0,4 & 99,6 \\
14 & 1 & 0,2 & 99,8 \\
15 & 1 & 0,2 & 100 \\
\hline & & & \\
\hline
\end{tabular}

En cuanto a los estadísticos descriptivos para la puntuación total de la escala, cabe señalar que la media global del POSIT $_{\text {UAS }}$ es de 1,52 y la desviación típica 2,34, con un rango de puntuaciones observado entre 0 y 15 . Los estadísticos de asimetría y curtosis estandarizados revelan la existencia de una marcada asimetría positiva $(A=21,36)$ y una distribución leptocúrtica $(C=28,10)$, lo cual pone de manifiesto que las puntuaciones no siguen una distribución normal. La ausencia de la normalidad también se ve reflejada en la distribución de los percentiles. Concretamente, el percentil 95 se corresponde con una puntuación de 6,5 (por debajo del recorrido medio de la escala). El incumplimiento de normalidad fue verificado mediante la prueba de Kolmogorov-Smirnov, con la correspondiente corrección de Lilliefors (K-S = 0,26; $p<, 001)$.

La tabla 2 recoge la distribución de frecuencias y los porcentajes acumulados para las diferentes puntuaciones. Si se adopta el punto de corte original de la escala $(\geq 2)$ es posible señalar que un $32,9 \%$ de la muestra presenta un resultado positivo en el POSIT $_{\text {UAS }}$.

Cuando se comparan las puntuaciones medias por género, se observa que si bien las mujeres obtienen una puntuación inferior a la de los hombres $(1,40$ vs 1,62$)$ esta diferencia no llega a ser estadísticamente significativa $\left(t_{(563)}\right.$ $=1,15 ; p=, 25)$. Por lo que se refiere a la edad, las diferencias entre los tres grupos establecidos (12-14 años, 15-16 y $17-18)$ sí son estadísticamente significativas $\left(\mathrm{F}_{(2-566)}=80,44\right.$; $\left.p<, 001 ; \eta_{\mathrm{p}}^{2}=0,22\right)$, siendo el grupo de 17-18 años el que presenta un promedio mayor $(3,13)$, seguido del de $15-16$ $(2,02)$ y el de $12-14(0,41)$.

\section{Consistencia de las puntuaciones}

Como evidencia de la fiabilidad del POSIT $_{\text {UAS }}$ se calculó su consistencia interna. Dado el carácter dicotómico de los ítems, ésta fue evaluada mediante el cálculo del índice KR20, obteniéndose un valor a de ,82. Pardo y Ruiz (2001) señalan que los valores por encima de ,80 se suelen considerar meritorios. Se analizó también la consistencia de cada ítem de forma individual, a través del cálculo del Índice de Homogeneidad Corregido (IHC), obteniendo valores entre 20 y ,60. Los ítems 1 y 16 fueron los que se mostraron menos consistentes con el conjunto de la escala. Sin embargo, la eliminación de ninguno de ellos reportaba mejora alguna sobre la consistencia global de la escala (Tabla 3).

\section{Evidencias de validez de estructura interna}

Una cuestión muy poco estudiada en la literatura es la dimensionalidad del POSIT $_{\mathrm{UAS}}$. La mayor parte de los trabajos ofrecen una puntuación global que es interpretada en base a un punto de corte, asumiendo el carácter unidimensional de la escala, avalado por su elevada consistencia interna. En el presente trabajo se ha intentado dar un paso más en ese sentido, realizando un Análisis Factorial Confirmatorio (AFC) que aportase evidencias de su validez interna, corroborando su estructura unifactorial. Los índices de bondad de ajuste obtenidos (GFI, Goodness of Fit Index; AGFI, Adjusted Goodness of Fit Index y NFI, Normed Fit Index), presentaron valores muy aceptables $(\mathrm{GFI}=, 983$; $\mathrm{AGFI}=$ ,978 y NFI = ,962) acordes a los criterios recogidos por Byr-

Tabla 3. Consistencia de los ítems del POSIT UAS.

\begin{tabular}{|c|c|c|}
\hline Item & $\begin{array}{l}\text { KR-20 } \\
\text { si se elimina el ítem }\end{array}$ & IHC \\
\hline 1 &, 822 & ,204 \\
\hline 2 & ,824 & ,362 \\
\hline 3 & ,810 & ,468 \\
\hline 4 & ,812 & ,452 \\
\hline 5 & ,810 &, 478 \\
\hline 6 & ,810 &, 513 \\
\hline 7 & ,807 & ,497 \\
\hline 8 & ,812 & ,466 \\
\hline 9 & ,819 & ,322 \\
\hline 10 & ,819 & ,337 \\
\hline 11 & ,801 &, 565 \\
\hline 12 & ,802 &, 562 \\
\hline 13 & ,804 &, 540 \\
\hline 14 & ,814 & ,395 \\
\hline 15 & ,798 & ,602 \\
\hline 16 & ,820 & ,260 \\
\hline 17 & ,811 & ,461 \\
\hline GLOBAL & 0,821 & \\
\hline
\end{tabular}


ne (2009) y Kline (2005), siendo además prácticamente idénticos en el caso de hombres $\left(\mathrm{GFI}_{\mathrm{H}}=, 979 ; \mathrm{AGFI}_{\mathrm{M}}=, 973\right.$ y $\left.\mathrm{NFI}_{\mathrm{M}}=, 958\right)$ y de mujeres $\left(\mathrm{GFI}_{\mathrm{M}}=, 977 ; \mathrm{AGFI}_{\mathrm{M}}=, 971 \mathrm{y}\right.$ $\mathrm{NFI}_{\mathrm{M}}=$,944). Se llevó a cabo, no obstante, un análisis de invarianza factorial siguiendo las pautas de Byrne (2009), encontrando que la comparación entre los diferentes modelos o niveles de restricción sugiere una misma estructura factorial para chicos y chicas en lo que se refiere a las saturaciones factoriales $\left(\Delta c^{2}=24,79 ; p=, 13\right)$, aunque no así respecto a los errores de medida $\left(\Delta c^{2}=126,78 ; p<, 001\right)$.

\section{Sensibilidad, especificidad y Curva ROC}

Los valores de sensibilidad y especificidad para diferentes puntos de corte se recogen en la tabla 4 . Como se puede observar, el punto de corte 2 es el que alcanza un mayor equilibrio entre los valores de sensibilidad (94,3\%) y especificidad $(83,9 \%)$. En otras palabras, la subescala es capaz de detectar verdaderos positivos en el 94,3\% de los casos y de rechazar verdaderos negativos en el $83,9 \%$, siendo ambos resultados muy aceptables. De manera complementaria, se llevó a cabo un Análisis de Curva ROC (Receiver Operating Characteristic), alcanzando un área bajo la curva de, 95 (Figura 1).

Si se adopta el "2" como punto de corte y se analizan las propiedades psicométricas del POSIT $_{\mathrm{UAS}}$ atendiendo al género, se puede observar que los resultados son muy similares, aunque en el caso de las mujeres se ve penalizada ligeramente la especificidad de la escala $(80,4 \%)$. En cuanto a la edad, los resultados son aceptables en los tres grupos considerados, especialmente en el de 12-14 años ( sensibilidad $=100 \%$ y especificidad $=94,4 \%)$, empeorando la especificidad en los dos restantes.

Tabla 4. Propiedades psicométricas del POSIT UAS para diferentes puntos de corte.

\begin{tabular}{lllll}
\hline & & $\begin{array}{l}\text { Sensibilidad } \\
(\%)\end{array}$ & $\begin{array}{l}\text { Especificidad } \\
(\%)\end{array}$ & CURVA ROC \\
\hline Corte $\geq 1$ & 99,2 & 64,2 &, 953 & \\
Corte $\geq 2$ & 94,3 & 83,9 & & \\
Corte $\geq 3$ & 79,5 & 93,5 & & \\
Corte $\geq 4$ & 66,4 & 97,3 & & \\
& & & Sensibilidad & Especificidad \\
$(\%)$ & CURVA ROC \\
& & $(\%)$ & Corte $\geq 2$ & \\
& & & 86,7 &, 963 \\
Género & Hombres & 94,8 & 80,4 &, 938 \\
& Mujeres & 93,3 & 94,4 &, 997 \\
& $12-14$ años & 100 & 73 &, 917 \\
\hline
\end{tabular}

\section{Evidencias de validez de relación con variables externas}

Para estudiar la validez de criterio se comparó, en primer lugar, el porcentaje de adolescentes que dieron positivo en el POSIT $_{\text {UAS }}$ y en el ADI $(32,9 \%$ y $21,4 \%$, respectivamente), obteniéndose un índice Kappa de concordancia de ,66 $(p<, 001)$. En segundo lugar, se realizó esa misma comparación entre el POSIT $_{\text {UAS }}$ y el CRAFFT, siendo el porcentaje de positivos en esta última herramienta del 22,8\%, con un índice de concordancia Kappa de ,63 $(p<$ ,001). Por último, se calculó también el coeficiente de correlación de Pearson entre las puntuaciones del CRAFFT

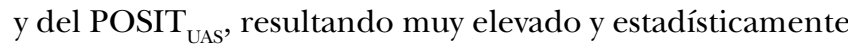
significativo $\left(\mathrm{r}_{\mathrm{xy}}=, 80 ; p<, 001\right)$.

\section{Discusión}

La adolescencia es un período crítico en el que los jóvenes típicamente se inician y experimentan con sustancias psicoactivas (Gonzálvez, Espada, Guillén-Riquelme, Secades y Orgilés, 2016). En España el consumo abusivo de alcohol y otras drogas entre los menores se ha convertido en uno de los principales problemas en términos de salud pública, tal y como se recogía en la Estrategia Nacional sobre Drogas 2009-2016 (Plan Nacional sobre Drogas, 2009b). Según los expertos el éxito de las políticas de prevención pasa en buena medida por favorecer la detección precoz de casos de riesgo, así como la realización de un seguimiento regular de los niveles detectados.

A pesar de que el POSIT constituye una de las herramientas de evaluación y screening a la que más suelen recurrir profesionales e investigadores del ámbito de las con-

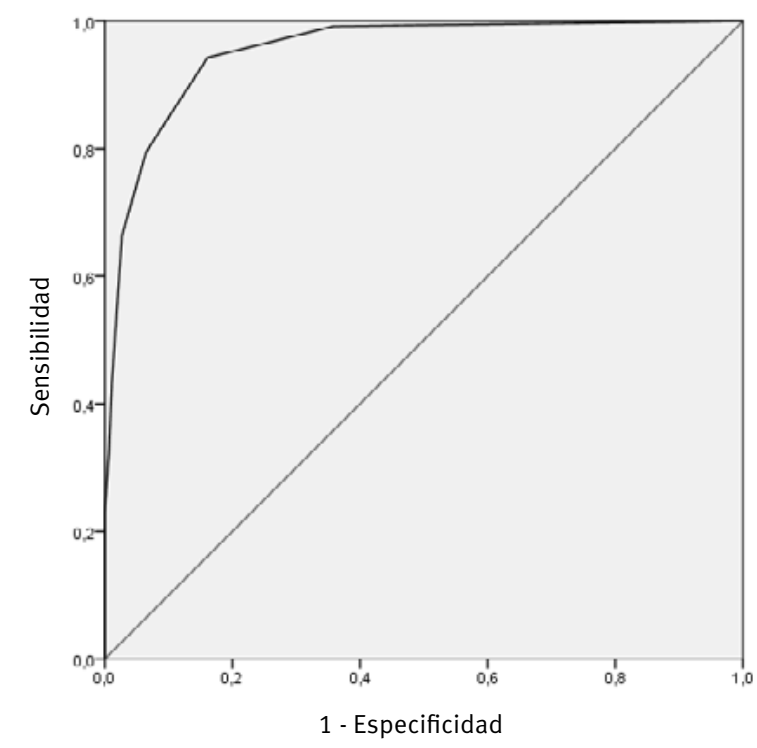

Figura 1. Curva ROC del POSIT UAS. 
ductas adictivas de todo el mundo, a día de hoy en España seguimos sin disponer de datos psicométricos concretos que nos permitan hacer uso de este instrumento con ciertas garantías.

Los análisis realizados a partir de una muestra de 569 estudiantes de la comunidad gallega permiten constatar que

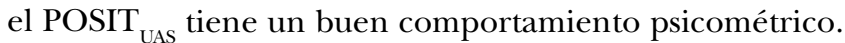
En primer lugar su consistencia interna es realmente elevada $(\alpha=, 82)$, en la línea de lo encontrado en otros trabajos de validación que coinciden en señalar que el POSIT $_{\text {UAS }}$ es una de las herramientas más consistentes (Knight et al., 2001; Mariño, González-Forteza, Andrade y Medina-Mora, 1998).

En segundo lugar, a nivel de cribado, muestra un meritorio equilibrio entre sensibilidad $(94,3 \%)$ y especificidad $(83,9 \%)$, para el punto de corte "2" señalado en la literatura. Además los resultados obtenidos por segmentos ponen de manifiesto que el POSIT $_{\text {UAS }}$ presenta un excelente comportamiento tanto en chicos como en chicas, al igual que entre diferentes grupos de edad, si bien se detecta una pérdida de especificidad cuando la edad aumenta, lo cual sugeriría elevar posiblemente los puntos de corte. No obstante, es importante hacer notar, tal y como señalaban Latimer et al. (1997), que ante una herramienta de screening lo más importante es maximizar la sensibilidad, habida cuenta de que la prioridad de este tipo de instrumentos es evitar que un adolescente con abuso de drogas sea omitido en el screening (falso negativo).

La elevada sensibilidad del POSIT $_{\text {UAS }}$ se hace también manifiesta cuando se compara el porcentaje de positivos obtenidos con esta herramienta $(32,9 \%)$ con los del CRAFFT $(22,8 \%)$, tal y como ya se señalaba en el trabajo de Golpe et al. (2016). Aun así el hecho de encontrar una elevada concordancia tanto con el CRAFFT como con el ADI, proporciona evidencias de su elevada validez de criterio.

Respecto a la dimensionalidad de la subescala, a pesar de ser una cuestión poco abordada en la literatura, la realización de un AFC ha permitido constatar que posee una estructura unifactorial, lo cual constituye un nuevo aval para trabajos como el de Knight et al. (2001), Latimer et al. (1997), Mariño et al. (1998) y Rumpf et al. (2013) en los que se asume de manera implícita dicha unidimensionalidad.

Como consecuencia de los resultados obtenidos es posible confirmar el satisfactorio comportamiento psicométrico de la subescala de Uso y Abuso de Sustancias $\left(\right.$ POSIT $\left._{\text {UAS }}\right)$, que puede ser utilizada con garantías por investigadores y profesionales españoles del ámbito de las conductas adictivas.

Desde un punto de vista aplicado los resultados obtenidos en el presente trabajo podrían conllevar algunas implicaciones de interés. En primer lugar, disponer de la validación del POSIT $_{\text {UAS }}$ en adolescentes españoles resulta interesante en la medida en que puede ser incorporado en futuras ediciones del principal sistema de información oficial del que disponemos en España respecto al consumo de sustancias en enseñanzas secundarias, el ESTUDES. En segundo lugar, contar con un instrumento validado de detección precoz de consumo de riesgo de alcohol y otras drogas en adolescentes como es el POSIT $_{\text {UAS }}$ podría servir de base para el desarrollo en España de un sistema de detección precoz e intervención breve, similar al SBIRT (Screening, Brief Intervention and Referal to Treatment), existente en los Estados Unidos de América (Laespada, 2014). Se trataría éste de un procedimiento que permitiese detectar, prevenir, intervenir y derivar a tratamiento a adolescentes con problemas por el uso de sustancias. Por último, contar con herramientas de cribado debidamente adaptadas y validadas en nuestro país contribuiría a una evaluación más completa y objetiva de los programas de prevención puestos en marcha, así como a un seguimiento regular de los niveles de consumo de riesgo detectados.

No obstante, es importante señalar algunas limitaciones del presente estudio. Desde el punto de vista muestral cabe destacar que a pesar de que se utilizaron casi 600 adolescentes (tamaño muestral similar o incluso superior al de otros estudios de validación) (Knight et al., 2001, 2003; Latimer et al., 1997), resulta insuficiente para realizar una baremación del instrumento atendiendo a diferentes segmentos sociodemográficos. Por otra parte, el hecho de que sólo se hayan utilizado adolescentes de la comunidad autónoma de Galicia constituye en sí mismo un condicionante de la validez externa. A pesar de que se han incluido estudiantes tanto de centros públicos como privados y concertados, residentes tanto en entornos urbanos como rurales o semirurales, es obvio que futuros trabajos han de marcarse como objetivo analizar el comportamiento psicométrico de la subescala en otras comunidades.

Hubiese sido de interés también poder disponer de información muestral acerca de algunos aspectos clínicos, como la presencia de diagnóstico comórbido, la existencia de historia familiar de trastorno, etc. Sin embargo, conviene advertir que se trata de un primer estudio de validación en el ámbito escolar, contexto en el que pretende ser utilizado el instrumento de forma más inmediata.

Por último, el hecho de que los datos no hayan sido recogidos mediante una entrevista clínica como tal, en el entorno de los servicios de salud, sino en el contexto escolar, hace que todas las variables hayan sido autoinformadas, por lo que es imposible conocer a ciencia cierta en qué medida los adolescentes pueden realmente haber infraestimado o sobreestimado sus niveles de consumo. No obstante, como han señalado previamente diferentes expertos del ámbito de las conductas adictivas, las medidas de autoinforme han demostrado ser fiables e incluso mejores que otros métodos a la hora de evaluar los niveles de consumo de alcohol y otras drogas (Babor, Kranzler y Lauerman, 1989; Winters, Stinchfield, Henly y Schwartz, 1990). 


\section{Reconocimientos}

Los autores de este trabajo quieren agradecer la financiación recibida a través de la Delegación del Gobierno para el Plan Nacional sobre Drogas (Ref. 2013/046) para la realización de este estudio.

\section{Conflicto de intereses}

Los autores de este artículo declaran no tener ningún conflicto de intereses

\section{Referencias}

American Psychiatric Association [APA]. (2013). Diagnostic and statistical manual of mental disorders (fifth edition) (DSM-5). Washington, DC: American Psychiatric Publishing.

Araujo, M., Harris, S.K., Knight, J.R., Gómez, P., Varela, J., Braña, T. y Lamas, M.J. (septiembre, 2015). CRAFFT validation study in a Spanish sample: Psychometric properties and recommendations. Trabajo presentado en el First European conference on addictive behaviours and dependencies, Lisboa, Portugal.

Arbuckle, J. L. (2012). AMOS 21.0. Crawfordville, FL: Amos Development Corporatio

Babor, T. F., Kranzler, H. R. y Lauerman, R. J. (1989). Early detection of harmful alcohol consumption: comparison of clinical, laboratory, and self-report screening procedures. Addictive Behaviors, 14, 139-157. doi:10.1016/03064603(89)90043-9.

Bobes, M. T., García, M. P., Marín, M., Ponce, G., Martínez, I. y Rubio, G. (2013). Detección y diagnóstico del trastorno por consumo de alcohol. En F. Pascual, J. Guardia, C. Pereiro y J. Bobes (Eds.), Alcoholismo: guía de intervención en el trastorno por consumo de alcohol (pp. 99-138). Barcelona, España: Grupo SANED.

Byrne, B. M. (2009). Structural equation modeling with AMOS: Basic concepts, applications, and programming (2. ${ }^{\mathrm{a}}$ ed.). London: Psychology Press.

Center for Substance Abuse Treatment. (2012). Screening and assessment of alcohol and other drug abusing adolescents. Treatment Improvement Protocol (TIP) - $N^{\circ}$ 31. Rockville, MD: Substance Abuse and Mental Health Services Administration, DHHS Publication.

Dembo, R., Schmeidler, J., Borden, P., Turner, G., Sue, C. y Manning, D. (1996). Examination of the reliability of the Problem Oriented Screening Instrument for Teenagers (POSIT) among arrested youths entering a juvenile assessment center. Substance Use E् Misuse, 31, 785-824. doi:10.3109/10826089609063958.

European Monitoring Centre for Drugs and Drug Addiction (EMCDDA). (1998). The Problem Oriented Screening Instrument for Teenagers (POSIT) - Revised Spanish Langua- ge Version. Recuperado de http://www.emcdda.europa. $\mathrm{eu} / \mathrm{html} . \mathrm{cfm} /$ index4439EN.html.

Farke, W. y Anderson, P. (2007). El consumo concentrado de alcohol en Europa. Adicciones, 19, 333-340.

Fuller, P. y Cavanaugh, R. (1995). Basic assessment and screening for substance abuse in the pediatrician's office. Pediatrics Clinics of North America, 42, 295-315. doi:10.1016/ S0031-3955(16)38948-9.

García, M.A., Novalbos, J.P., Martínez, J.M. y O’Ferrall, C. (2016). Validación del test para la identificación de trastornos por uso de alcohol en población universitaria: AUDIT y AUDIT-C. Adicciones, 28, 194-204.

Golpe, S., Araujo, M., Harris, S.K., Knight, J.R., Gómez, P. y Rial, A. (junio, 2016). Detección precoz del consumo de alcohol y otras drogas en adolescentes: validación empírica del CRAFFT en la comunidad gallega. Trabajo presentado en el III Congreso de Psicoloxía Profesional de Galicia, Santiago de Compostela, España.

Gonzálvez, M.T., Espada, J.P., Guillén-Riquelme, A., Secades, R. y Orgilés, M. (2016). Asociación entre rasgos de personalidad y consumo de sustancias en adolescentes españoles. Adicciones, 28, 108-115. doi:10.20882/adicciones. 777 .

Huang, J. H., Jacobs, D. F. y Deverensky, J. L. (2010). Sexual risk-taking behaviors, gambling, and heavy drinking among U.S. college athletes. Archives of Sexual Behavior, 39, 706-713. doi:10.1007/s10508-009-9521-7.

IBM Corp. Released (2011). IBM SPSS Statistics for Windows, Version 20.0. Armonk, NY: IBM Corp.

Jones, S. E., Oeltmann, J., Wilson, T. W., Brener, N. D. y Hill, C. V. (2001). Binge drinking among undergraduate college students in the United States: Implications for other substance use. Journal of American College Health, 50, 33-38. doi:10.1080/ 07448480109595709.

Jöreskog, K. G. y Sörbom, D. (1989). LISREL 7 User's reference guide. Chigaco: Scientific Software.

Kline, R. B. (2005). Principles and Practice of Structural Equation Modeling (2. ${ }^{\mathrm{a}}$ ed.). New York: The Guilford Press.

Knight, J. R., Goodman, E., Pulerwitz, T. y DuRant, R. (2001). Reliability of the Problem Oriented Screening Instrument for Teenagers (POSIT) in adolescent medical practice. Journal of the Adolescent Health, 29, 125-130. doi:10.1016/S1054-139X(00)00206-8.

Knight, J. R., Sherritt, L., Harris, S., Gates, E. y Chang, G. (2003). Validity of brief alcohol screening tests among adolescents: A comparison of the AUDIT, POSIT, CAGE and CRAFFT. Alcoholism: Clinical E Experimental Research, 27, 67-73. doi:10.1111/ j.1530-0277.2003.tb02723.x.

Knight, J. R., Sherritt, L., Shrier, L. A., Harris, S. K. y Chang, G. (2002). Validity of the CRAFFT Substance Abuse Screening Test among adolescent clinical patients. Archives of Pediatrics $\mathcal{E}$ Adolescent Medicine, 156, 607-614. doi:10.1001/archpedi.156.6.607. 
Knight, J. R., Shrier, L. A., Bravender, T. D., Farrell, M., Vander Bilt, J. y Shaffer, H. J. (1999). A new brief screen for adolescent substance abuse. Archives of Pediatrics $\mathcal{E}^{\circ}$ Adolescent Medicine, 153, 591-596. doi:10.1001/archpedi.153.6.591.

Kuder, G. F. y Richardson, M. W. (1937). The theory of the estimation of test reliability. Psychometrika, 2, 151-160.

Laespada, M.T. (2014). Consumo de alcohol en jóvenes y adolescentes. Una mirada ecológica. Bilbao, España: Universidad de Deusto, Instituto de Deusto de Drogodependencias.

Latimer, W., O’Brien, M., McDouall, J., Toussova, O., Floyd, L. y Vazquez, M. (2004). Screening for «substance abuse» among school-based youth in Mexico using the Problem Oriented Screening Instrument (POSIT) for Teenagers. Substance Use $\mathcal{E}$ Misuse, 39, 307-329. doi:10.1081/JA-120028492.

Latimer, W., Winters, K. C. y Stinchfield, R. D. (1997). Screening for drug abuse among adolescents in clinical and correctional settings using the Problem Oriented Screening Instrument for Teenagers. The American Journal of Drug and Alcohol Abuse, 23, 79-98. doi:10.3109/00952999709001689.

Legleye, S., Piontek, D. y Kraus, L. (2011). Psychometric properties of the Cannabis Abuse Screening Test (CAST) in a French sample of adolescents. Drug and Alcohol Dependence, 113, 229-235. doi:10.1016/j.drugalcdep.2010.08.011.

López-Caneda, E., Mota, N., Crego, A., Velasquez, T., Corral, M., Rodríguez, S. y Cadaveira, F. (2014). Anomalías neurocognitivas asociadas al consumo intensivo de alcohol (binge drinking) en jóvenes y adolescentes: una revisión. Adicciones, 26, 334-359. doi:10.20882/adicciones.39.

Mariño, M., González-Forteza, C., Andrade, P. y Medina-Mora, M. (1998). Validación de un cuestionario para detectar adolescentes con problemas por el uso de drogas. Salud Mental, 21, 27-36.

Matali, J.L., Andión, O., Pardo, M., Iniesta, R., Serrano, E. y San, L. (2016). Adolescentes y diagnóstico dual en el servicio de urgencias psiquiátricas. Adicciones, 28, 71-79. doi:10.20882/adicciones.783.

McLaney, M., Del Boca, F. y Babor, T. (1994). A validation study of the Problem Oriented Screening Instrument for Teenagers (POSIT). Journal of Mental Health, 3, 363376. doi:10.3109/09638239408997947.

McPherson, T. y Hersch, R. (2000). Brief substance use screening instruments for primary care settings: A review. Journal of Substance Abuse Treatment, 18, 193-202. doi:10.1016/S0740-5472(99)00028-8.

Miller, J. W., Naimi, T. S., Brewer, R. D. y Jones, S. E. (2007). Binge drinking and associated health risk behaviors among high school students. Pediatrics, 119, 76-85. doi:10.1542/ peds.2006-1517.
Motos, P., Cortés, M. T., Giménez, J. A. y Cadaveira, F. (2015). Predictores del consumo semanal de alcohol y sus consecuencias asociadas en universitarios consumidores intensivos de alcohol. Adicciones, 27, 119-131.

Organización Mundial de la Salud. (1997). Working group on the identification and management of psychoactive substance use problems in primary health care settings. San Francisco, CA: Division on Mental Health and Prevention of Substance Abuse.

Parada, M., Corral, M., Caamaño-Isorna, F., Mota, N., Crego, A., Rodríguez Holguín, S. y Cadaveira, F. (2011). Definición del concepto de consumo intensivo de alcohol adolescente (binge drinking). Adicciones, 23, 53-63.

Pardo, A. y Ruiz, M. A. (2001). SPSS 11. Guía para el análisis de datos. Madrid: McGraw-Hill.

Petit, G., Maurage, P., Kornreich, C., Verbanck, P. y Campanella, S. (2014). Binge drinking in adolescents: A review of neurophysiological and neuroimaging research. Alcohol and Alcoholism, 49, 198-206. doi:10.1093/alcalc/agt172.

Plan Nacional sobre Drogas. (2009a). Plan de Acción sobre Drogas, España 2013-2016. Madrid, España: Delegación del Gobierno para el Plan Nacional sobre Drogas, Ministerio de Sanidad, Servicios Sociales e Igualdad.

Plan Nacional sobre Drogas. (2009b). Estrategia Nacional sobre Drogas 2009-2016. Madrid, España: Delegación del Gobierno para el Plan Nacional sobre Drogas, Ministerio de Sanidad y Consumo.

Plan Nacional sobre Drogas. (2016). Encuesta sobre el uso de drogas en enseñanzas secundarias en España (ESTUDES) 2014-2015. Madrid, España: Delegación del Gobierno para el Plan Nacional sobre Drogas, Ministerio de Sanidad, Servicios Sociales e Igualdad.

Rahdert, E. R. (1991). The Adolescent Assessment/Referral System. Rockville, MD: National Institute on Drug Abuse.

Rumpf, H., Wohlert, T., Freyer-Adam, J., Grothues, J. y Bischof, G. (2013). Screening questionnaires for problem drinking in adolescents: Performance of AUDIT, AUDIT-C, CRAFFT and POSIT. European Addiction Research, 19, 121-127. doi:10.1159/000342331.

Tiburcio Sainz, M., Rosete-Mohedano, M.G., Natera Rey, G., Martínez Vélez, N.A., Carreño García, S. y Pérez Cisneros, D. (2016). Validez y confiabilidad de la prueba de detección de consumo de alcohol, tabaco y sustancias (ASSIST) en estudiantes universitarios. Adicciones, 28, 19-27. doi:10.20882/adicciones.786.

Timken, D. S. (2007). Colorado Alcohol and Drug Abuse Division (ADAD) approved evaluation instrumentation. Boulder, CO: Colorado Department of Human Services, Alcohol and Drug Abuse Division.

Windle, M. (2003). Alcohol use among adolescents and young adults. Alcohol Research and Health, 27, 79-85.

Winters, K. C. y Henly, G. (1993). Adolescent Diagnostic Interview (ADI). Los Ángeles, CA: Western Psychological Services. 
Winters, K. C., Stinchfield, R. D., Henly, G. A. y Schwartz, R. H. (1990). Validity of adolescent self-report of alcohol and other drug involvement. International Journal of the Addictions, 25, 1379-1395. doi:10.3109/10826089009068469.

Xunta de Galicia. (2010). Plan de Trastornos Adictivos de Galicia 2011-2016. Santiago de Compostela, España: Consellería de Sanidade, Xunta de Galicia. Recuperado de http://xuventude.xunta.es/uploads/docs/ Observatorio/Plan_de_trastornos_adictivos_de_Galicia_2011-2016.pdf. 